\title{
Éléments pour une prospective du milieu
} L'enjeu du sensible en aménagement

\section{Toward a Prospective View of Milieu. Facing the Challenge of the Sensitive Dimension in Land Development Elementos para une prospectiva del medio ambiente. La importancia de lo sensible en planeamiento}

\section{Olivier Labussière}

Volume 54, numéro 153, 2010

URI : https://id.erudit.org/iderudit/1005608ar

DOI : https://doi.org/10.7202/1005608ar

\section{Aller au sommaire du numéro}

Éditeur(s)

Département de géographie de l’Université Laval

ISSN

0007-9766 (imprimé)

1708-8968 (numérique)

Découvrir la revue

Citer cet article

Labussière, O. (2010). Éléments pour une prospective du milieu : l'enjeu du sensible en aménagement. Cahiers de géographie du Québec, 54(153), 499-515. https://doi.org/10.7202/1005608ar
Résumé de l'article

Cet article pose la question du sensible en aménagement en tentant de voir en quoi, au-delà du registre de l'expérience, elle pourrait contribuer à une réflexion sur les normes de ses modèles d'action, leurs points de blocage et d'innovation. En rapprochant l'aménagement d'une activité clinique ouverte à l'expérimentation et favorisant un usage non instrumental des normes, nous procédons à un déplacement de la question du sensible d'une caractéristique associée au milieu à un mode de relation (cognitif, politique, expérientiel) de l'aménagement avec le milieu. La façon dont le sensible est configuré et distribué décide en partie de la normativité de la démarche aménagiste, de son ouverture à la logique du sujet et de la capacité de celui-ci à donner à son mode d'habiter, comme à lui-même, une actualité qui corresponde aux enjeux de son époque. Nous resituons cette réflexion, et les propositions qui la sous-tendent, à l'égard d'une problématique contemporaine, celle de la transition énergétique et de la planification des paysages éoliens. 


\title{
Éléments pour une prospective du milieu L'enjeu du sensible en aménagement
}

\author{
Toward a Prospective View of Milieu. \\ Facing the Challenge of the Sensitive \\ Dimension in Land Development \\ Elementos para une prospectiva del medio \\ ambiente. La importancia de lo sensible en \\ planeamiento
}

\author{
Olivier LABUSSIÈRE \\ Centre International de Recherche sur \\ I'Environnement et le Développemen (CIRED) \\ Olivier.Labussiere@centre-cired.fr
}

Résumé

Cet article pose la question du sensible en aménagement en tentant de voir en quoi, au-delà du registre de l'expérience, elle pourrait contribuer à une réflexion sur les normes de ses modèles d'action, leurs points de blocage et d'innovation. En rapprochant l'aménagement d'une activité clinique ouverte à l'expérimentation et favorisant un usage non instrumental des normes, nous procédons à un déplacement de la question du sensible d'une caractéristique associée au milieu à un mode de relation (cognitif, politique, expérientiel) de l'aménagement avec le milieu. La façon dont le sensible est configuré et distribué décide en partie de la normativité de la démarche aménagiste, de son ouverture à la logique du sujet et de la capacité de celui-ci à donner à son mode d'habiter, comme à lui-même, une actualité qui corresponde aux enjeux de son époque. Nous resituons cette réflexion, et les propositions qui la sous-tendent, à l'égard d'une problématique contemporaine, celle de la transition énergétique et de la planification des paysages éoliens.

\section{Mots-clés}

Pensée clinique, sensible, aménagement, milieu géographique, sujet.

\begin{abstract}
In this article we investigate the role of the sensitive dimension in land development as we attempt to assess its potential contribution to a deliberation on the normativity of its action models, and their sticking points and capacity to innovate. Initially, land development is compared to a clinical activity that encourages experimentation as well as a non-instrumental application of these norms; and the sensitive dimension of features associated with the geographical milieu is transferred and integrated into a cognitive, political and experiential relationship between land development and milieu. The way the sensitive dimension is configured and distributed partially dictates the normativity of land development, its receptivity to the logic of the individual subject and his/her capacity to bring to his/her mode of living and himself/herself a topicality reflecting today's challenges. Finally, we shift our focus from this deliberation on a contemporary issue and the propositions on which it is based to the subject of energy transfer and development of landscapes dominated by windmills.
\end{abstract}

\section{Keywords}

Clinical thought, sensitive dimension, land development, geographical milieu, individual subject. 


\section{Resumen}

Este artículo plantea la cuestión de lo sensible en planeamiento, y allende el registro de la experiencia, trata de ver como contribuiría a une reflección normativa sobre los modelos de acción, sus puntos de bloqueo y de inovación. Asociando planeamiento y clínica experimental, sin instrumentalizar las normas, trasladamos la cuestión de lo sensible de una característica asociada al medio, a un modo de relación (cognoscitivo, político, experiencial) del planeamiento con el medio ambiente. La configuración y la distribución de lo sensible decide, en parte, la normatividad de la gestión planificadora, la comprensón de la lógica del sujeto, de la capacidad de éste de dar, y dase a sí mismo, la actualidad de los invites de su época. Esta reflexión, y las proposiciones subyacentes son discutidas dentro de una problemática contemporánea, la de la transición energética y de la planificación de paisajes eólicos.

\section{Palabras claves}

Pensamiento clínico, lo sensible, planeamiento, medio geográfico, sujeto.

a prise en compte des milieux géographiques en aménagement est une opération délicate: elle se réduit souvent à une expertise environnementale qui tend à segmenter ce qui est vécu comme un tout et qui se focalise sur les impacts d'un projet, dans une optique gestionnaire. Pour dépasser cette approche instrumentale, une façon de procéder consiste à se tourner vers la question du sensible pour se saisir du milieu dans sa complexité (Berdoulay, 2000).

La question du sensible s'est déployée, ces dernières décennies, à la croisée de multiples champs disciplinaires: en géographie, avec les travaux sur l'espace vécu et le sujet (Berdoulay et Entrikin, 1998; Berque, 2000a; Tuan, 2006) ; dans le domaine du paysage, avec la remise en cause d'une approche strictement culturelle (Cosgrove, 1984; Roger, 1997) et le regain d'intérêt pour une lecture plus factuelle et située (Dewitte, 2001); dans le domaine esthétique, avec l'éloignement d'une approche contemplative et l'accent mis sur l'expérience quotidienne, l'action et la relation à l'environnement (Berleant, 1992; Kupfer, 2003). La vitalité de ces débats fait, depuis une décennie, de la question du sensible une entrée de plus en plus mobilisée pour étudier l'aménagement (Augoyard, 1995; Faburel, 2003 ; Amphoux et al., 2004; Lolive et Soubeyran, 2007; Blanc, 2008; Labussière, 2009a).

Toutefois, si elle met en valeur les liens entre l'homme et son milieu, sa capacité à dialoguer avec les démarches d'aménagement pose encore question. Le sensible, perçu comme ce qui a trait au registre de l'expérience, apparaît en décalage avec la rationalité aménagiste dont les modèles d'action sont fortement normés. Cela peut être illustré par le paradoxe suivant: l'entrée du sensible valorise les qualités singulières du milieu géographique au risque d'en faire un cas d'exception vis-à-vis duquel l'aménagement hésite à entreprendre des processus innovants et coûteux (Labussière, 2009a). Ces difficultés ne sont pas seulement relatives à la pratique aménagiste. Elles concernent aussi la façon dont nous articulons la question du sensible à la rationalité aménagiste, ainsi que la place accordée au sujet. 
Cet article propose d'étudier les conditions qui permettraient à la question du sensible d'assumer le rapport entre la norme et l'innovation en aménagement. Pour ce faire, nous examinons un modèle cognitif et pratique, celui de la clinique médicale, où l'attention pour le sensible contribue à ajuster l'usage des normes, voire à les renouveler, en fonction du cas. À travers ce prisme, il s'agit aussi d'apprendre sur les liens entre l'aménagement et la logique du sujet, en réinvestissant la question de la sensibilité de l'aménageur comme un trait essentiel de l'activité planificatrice (Berdoulay et Entrikin, 1994).

Cette perspective dessine un parcours en quatre étapes: (i) la pensée clinique introduit à une intelligence du sensible qui valorise l'expérimentation et la création, (ii) et suggère un usage non instrumental des normes, utile pour ouvrir l'aménagement aux milieux géographiques. Cette lecture (iii) confère au milieu une dimension de potentiel et éclaire la façon dont le sujet peut trouver son actualité à l'intérieur et à l'occasion des processus d'aménagement. Cette réflexion théorique se double d'une réflexion méthodologique (iv) en ce que la question du sensible ainsi posée permet de suivre les modalités cognitives, politiques et expérientielles mobilisées en planification (le cas de l'énergie éolienne) et d'analyser en quoi leur évolution renouvelle la prise en compte du milieu.

\section{Pensée clinique et aménagement: sentir, expérimenter, créer}

Dans la pensée aménagiste, la référence au monde médical est bien souvent fondatrice d'une approche instrumentale (Choay, 1980). Pensons, par exemple, à Le Corbusier endossant la blouse du chirurgien pour faire «respirer» Paris (Le Corbusier, 1994). La métaphore médicale remplit un usage polémique et normatif mais, subtile, elle offre une perspective naturaliste qui exclut la manipulation arbitraire (Schlanger, 1971).

Cette perspective, somme toute limitée, se déploie entre l'étiologie (l’identification des causes) et la thérapeutique (l'application d'un traitement). En ce sens, bien que répandue dans la pensée aménagiste, la référence au monde médical est le plus souvent fondatrice d'une problématique restreinte qui délaisse un moment amont, celui de l'observation du corps et de ses manifestations sensibles: la symptomatologie.

Pour notre étude, le moment et la pratique symptomatologiques sont intéressants à revisiter en ce qu'ils introduisent à une intelligence spécifique du sensible. Songeons, par exemple, au médecin qui explore l'état d'un corps en le frappant du bout des doigts : il fait résonner les organes et dirige son attention en fonction des sons gras, caverneux, mats... Les signes produits constituent la manifestation sensible de phénomènes non directement intelligibles. Ils placent l'observateur en position d'écoute. C'est donc une activité interprétative consistant à ausculter le corps et à relever ou produire des symptômes qui, rassemblés en un faisceau d'indices, permettent au médecin d'avancer l'hypothèse d'une maladie. Le raisonnement symptomatologique est de nature abductive.

Ce moment amont appelle à être précisé. Car la symptomatologie, outre qu'elle a connu des fortunes diverses, ne saurait être invoquée de façon générique. Foucault (2007) a fort bien montré que la clinique n'est pas une forme de «sentir» indifférenciée, un préalable immémorial entre le médecin et le malade. L’action de «sentir» répond à 
des pratiques cliniques distinctes. Au XVIII ${ }^{e}$ siècle, la "clinique des espèces ${ }^{1}{ }^{1}$ valorise la belle sensibilité du clinicien et fait du malade un exemplaire de sa maladie, le support d'une essence pathologique à connaître. Le malade n'est donc pas toujours le sujet de sa maladie; quant au sensible, la façon dont il est distribué confère parfois au médecin le pouvoir de dire une vérité du symptôme qui met à distance le malade.

Parmi les formes historiques de la clinique (Hutin, 2006; Foucault, 2007), la figure du médecin-musicien ${ }^{2}$ (Labussière, à paraître) est sans doute la plus proche de la perspective analytique élaborée dans cet article. Quelques traits permettent de préciser la valeur cognitive de cette symptomatologie: celle-ci mobilise l'ensemble des sens perceptifs; le sensible permet une connaissance rigoureuse de la maladie; les symptômes ne sont pas seulement observés, mais aussi produits grâce à l'introduction d'instruments techniques.

Cette symptomatologie excelle dans la description des modulations qu'une même maladie peut présenter chez différents individus ${ }^{3}$ et autorise donc une lecture différentielle des cas. L'individu n'est plus porteur d'une maladie générale, mais le sujet de sa maladie dont la forme modulée lui est propre. En s'appliquant à des individualités pathologiques, cette approche déploie un raffinement sémantique dans la description des manifestations sensibles (grain, texture, couleur, odeur, etc.). Le symptôme guide l'analyse autant qu'il pluralise le langage. L'expérimentation, autant corporelle (produire le signe) que verbale (le qualifier), prend l'allure d'un processus ouvert de désignation. Ce type d’intelligence clinique mérite toute notre attention.

Pour ce faire, une façon de procéder consiste à montrer les appuis qu'elle trouve dans la pensée contemporaine, en particulier celle de Gilles Deleuze. La symptomatologie intervient dans la pensée de l'auteur en rapport avec différentes traditions de pensée, celles de Nietzsche, de Spinoza ou encore de Kant.

La pensée nietzschéenne tient une place importante (Deleuze, 1962). C'est d'elle que provient l'idée d'ausculter les formes de la culture (religion, morale, politique, etc.) à la manière d'un médecin-musicien. Chez Nietzsche, plus que la pensée, c'est tout le corps dans son entier qui pense. Il est à la fois ce lieu premier, en prise avec la multiplicité sensible, et ce lieu «opaque» (Blondel, 1986: 291) qui ne livre aucune désignation directe. Il appelle donc une activité interprétative. L'interprétation est moins ici un exercice intellectuel qu'un corps à corps consistant à expérimenter différents modes de mise en relation avec la multiplicité. Deleuze réinvestit cette approche dans Marcel Proust et les signes (Deleuze, 2006). Il présente l'œuvre de Proust comme un roman

1 La clinique des espèces repose sur l'équivalence du voir et du savoir. La maladie, tout entière visible à la surface du corps, se manifeste par des signes dont l'inventaire permet de constituer la connaissance et d'identifier des pathologies fondamentales.

2 L'un des hérauts de cette posture clinique est René Laënnec (1781-1826). Auteur d'une thèse sur l'Ecole de Cos, Laënnec remet à l'honneur une pratique de l'auscultation largement inspirée par les textes hippocratiques et l'ouvre à de nouvelles méthodes. Il est l'inventeur du stéthoscope, instrument d'écoute à partir duquel il renouvela de façon considérable la sémiologie des troubles cardiaques et pulmonaires, consignée dans son célèbre traité De l'auscultation médiate (Laënnec, 1819).

3 Les symptômes modulés, loin de troubler la connaissance de la maladie, lui donnent une forme individuelle et expriment certains éléments liés au milieu social (Laënnec, 1819). Ces individualités pathologiques forment une galerie de portraits (l'artisan, la blanchisseuse, l'ancien soldat, le cordonnier...) à partir desquels s'organisent la mise en partage et la transmission du savoir clinique. 
d'apprentissage, une initiation aux signes. Le signe caractérise une expérience où le sensible force à penser. Il n'engage pas une entreprise de clarification du sens, mais un travail d'investigation.

Avec Spinoza, Deleuze met plus nettement l'accent sur l'expérimentation comme création. Le signe ne renvoie plus seulement au symptôme (logique médicale), il se double du registre de la sensation (logique esthétique) (Sauvagnargues, 2005). La sensation désigne ici un point de vue de compatibilité entre les choses. Le défi, autant esthétique qu'éthique, est d'inventer les codes permettant d'accéder et de former de nouvelles associations. Spinoza, nous dit Deleuze, propose un «art de l'Éthique»: «organiser les bonnes rencontres, composer les rapports vécus, former les puissances, expérimenter» (2003: 161). C'est là une forme d'évaluation créatrice qui rompt avec l'esprit d'expertise.

L'expérience singulière que constitue cette pensée par les signes sensibles est également présente chez Kant. La forme belle, l'être organisé font signes à l'homme (De Gramont, 1996). Ces signes ne disent rien de connaissable. La nature ne parle pas à l'homme. Mais sa réception sensible enjoint l'ouverture d'un langage - la finalité - et d'une forme de jugement - la réflexion. Cette forme d'intelligence, interprétative et inventive, tient à la capacité de l'homme d'être affecté. Deleuze (1997) la rapproche également du diagnostic médical.

Plus qu'une approche raisonnée du sensible, ces trois traditions de pensée introduisent à un renversement de l'ordre des facteurs : «en science et en philosophie, l'intelligence vient toujours avant; mais le propre des signes, c'est qu'ils font appel à l'intelligence en tant qu'elle vient après » (Deleuze, 2006: 120). Ce renversement tient au fait que le signe manifeste une réalité complexe, un état relationnel et sensible non réductible à nos catégories de pensée et d'action.

Vis-à-vis des enjeux de l'aménagement, cette approche clinique pose la question de la mise en forme de nos ignorances. Savons-nous de quoi les choses sont capables? Comment imaginer les multiples façons dont elles pourraient s'associer entre elles? Ce potentiel de relations n'est pas aisé à cerner. Il suppose d'inventer les méthodes garantissant l'exploration attentive des milieux géographiques dans lesquels ces relations émergentes trouvent leurs appuis. Cette posture est pour le moins inhabituelle en aménagement. Il est plus souvent question de réduire l'incertitude en procédant à l'évacuation du milieu - ce à quoi la pensée médicale n'est d'ailleurs pas étrangère (Soubeyran, 2000).

Par son ouverture au sensible et l'inventivité de langage qu'elle met en œuvre, cette approche clinique ouvre des pistes nouvelles pour revisiter les liens entre l'aménagement, le milieu et la logique du sujet. Encore faut-il préciser en quoi ce qui relève de l'expérimentation et de la création peut innerver l'action aménagiste. L’intérêt pour le sensible et la création est délicat à mobiliser dès lors qu'il s'agit de penser au-delà de l'expérience singulière d'un individu. L'aménagement en est presque un cas critique: il nous renvoie à des processus opérationnels fortement normés mettant en jeu, qui plus est, la production d'un espace commun. Aussi, pour mieux cerner le potentiel d'innovation des démarches d'aménagement, il est crucial de réfléchir sur leur caractère normatif. 


\section{Réfléchir sur la norme: une pensée de la «forme seconde »}

Depuis les années 1960, les démarches d'aménagement ont fait l'objet d'une profonde remise en cause, tant sur le plan théorique que pratique, pour leur rationalité trop normative et hiérarchique. Fondées, ces critiques ont motivé l'émergence d'une pensée alternative de la planification, allant parfois jusqu'à rejeter son principe au profit d'autres modèles d'action prétendument plus ouverts à l'expérimentation.

La pensée de Gilles Deleuze en ce qu'elle développe une critique des normes (de la pensée, du langage, de l'expérience...) peut être utile pour réfléchir sur l'aménagement sans tomber dans le piège consistant à opposer la planification et l'expérimentation, la norme et l'innovation. Sa réception en géographie, principalement dans des travaux anglo-saxons ${ }^{4}$ (Environment and Planning D, 1992 et 1996), l'a trop souvent réduite à une critique radicale de toute pensée normative. Nous revenons sur quelques-uns de ces travaux, puis proposons une autre lecture de la pensée deleuzienne à même d'ouvrir un dialogue entre l'aménagement et les milieux géographiques.

Les premiers articles consacrés aux travaux de Deleuze et Guattari en géographie apparaissent sous la plume de Marcus Doel. Sa lecture, centrée sur la perspective deleuzienne de la différence comme événement, illustre certains des excès évoqués. Par exemple, Doel (1993) rejette l'idée de différenciation spatiale qui réduit, selon lui, la question de la différence à une notion de séparation et, donc en galvaude le principe. Ceci le conduit à critiquer l'emploi de la notion de place, qu'il juge trop fixiste, et à promouvoir une pensée de flux non localisables (Doel, 1999). Partant de cette perspective, Doel promeut une géographie centrée sur l'action, mais il peine à la décrire de façon effective. Peet (2006) raille cette pensée de l'innovation qu'il juge attentiste, consacrée à la réception de l'événement en dehors de tout contexte social. Cette approche de l'action est également problématique en ce que Doel (1996) l'établit hors de l'histoire par opposition au temps de l'événement.

Une autre approche de la pensée deleuzienne émane des travaux de Thrift qui s'en saisit lorsqu'il entreprend de forger une théorie de la pratique (1996). Ce programme de travail vise à valoriser l'expérimentation plutôt que la théorie, à étudier les pratiques dans leur contexte et à rediscuter la dualité de la représentation et de la réalité. De façon étonnante, Thrift mobilise, avec retenue, la pensée deleuzienne: il ne souhaite pas abandonner le présent au profit du futur, la fixité au profit du mouvement et adopter une pensée de la multiplicité qui se déploierait hors de toutes contraintes. S’il est plus réservé que Doel quant à l'usage de la pensée deleuzienne, il la réduit, tout comme lui, à une visée anti-structuraliste radicale. Les quelques fois où il la mobilise, Thrift la met à profit pour approcher l'action sous l'angle de la pratique quotidienne, en montrant que le corps en situation dispose de capacités d'agencement entre les choses. Le modèle sous-jacent est celui de la performance artistique (Thrift, 2000; Thrift et Dewsbury, 2000) où l'action s'élabore dans le temps de l'événement.

Pour sa part, Dewsbury propose une lecture plus nuancée: la pensée deleuzienne peut articuler l'expérience sensible aux formes constituées dans le temps de l'histoire

4 La pensée de Deleuze et Guattari est régulièrement absente des croisements entre philosophie et géographie (Gale et Olsson, 1979; Benoist et Merlini, 2001). Le travail de Antonioli (2003) constitue une exception, avec le récent recueil de Buchanan et Lambert (2006). 
(Dewsbury, 2000). Malgré cela, l'auteur élabore une réflexion sur l'action performative qu'il cantonne au registre de l'immédiateté et qui ne retient que ce qui intéresse la sensation.

Ces travaux, en théâtralisant la survenue de l'événement, restreignent les possibilités de dialogue avec la géographie comme avec l'aménagement. Nous proposons une lecture qui n'oppose pas l'innovation à la question des normes. En effet, la pensée deleuzienne suggère un usage non instrumental des normes qui peut être utile pour ouvrir l'action aménagiste aux situations et réfléchir sur des formes d’interventions collectivement négociables.

Si Deleuze nous invite à expérimenter le dépassement des normes (du langage, de la pensée, de l'expérience), cela s'accompagne tout au long de son œuvre d'appels à la prudence (Deleuze et Guattari, 1980). Il semble même que les dimensions d'expérimentation et de création auxquelles il nous invite ne peuvent être comprises indépendamment de ce principe. «L'expérimentation, ça implique une prudence, le risque, c'est évidemment le contraire de se déstratifier, le risque suicidaire» (Deleuze, 1973: 12). Bien que rarement relevée, c'est là une objection notable adressée aux travaux qui assimilent la pensée deleuzienne à une pensée de flux et d'expérimentation sans limite. Il n'est pas question de se séparer définitivement des formes - la prudence exigerait plutôt d'en faire provision. «L'organisme [i.e. la forme], il faut en garder assez pour qu'il se reforme à chaque aube» (Deleuze et Guattari, 1980: 199).

Buydens (2005) est un des rares auteurs à avoir souligné cet aspect. Elle rappelle que la pensée deleuzienne s'organise autour de trois moments : dénoncer les découpages autoritaires du monde en objets, sujets, etc.; fluidifier les formes pour libérer leur multiplicité fondamentale; mettre en garde contre l'a-formel pur qui conduirait à la négation de la vie. Ainsi, Deleuze ne rejette pas ce qui est de l'ordre de la forme. Il dénonce sa pseudo-nécessité: «niée dans sa nécessité, rien ne s'oppose à ce qu'elle [la forme] soit réintroduite dans sa contingence» (Buydens, 2005 : 79). Cela ouvre la voie à une pensée de la «forme seconde».

Pour notre usage, la notion de «forme seconde» souligne deux aspects. Elle insiste sur l'importance pour une société de savoir entretenir un dialogue avec ses systèmes normatifs, y compris ses outils et ses méthodes d'aménagement de l'espace. La planification est régulièrement mise à l'index par les tenants d'une gouvernance libérale, lesquels souhaitent l'écarter du jeu économique et social plutôt que de renouveler l'intelligence avec laquelle elle pourrait être déployée. Il nous semble que la notion de «forme seconde» peut fournir un cadre conceptuel pour envisager les moyens d'un usage non instrumental des normes, et ce faisant, maintenir une réflexivité au cœur des processus collectifs de négociation des règles de production d'un espace commun. Outre la planification, c'est la relation de l'homme au milieu qui est plus largement mise en question. L'idée de «forme seconde» nous invite à ne pas réifier cette relation et à nous emparer avec prudence des processus historiques de mise en forme et de mise en sens de l'espace.

La notion de «forme seconde», et le principe de prudence qui la sous-tend, invitent par exemple à réfléchir sur les processus de qualification de l'espace pour retrouver en-deçà des zonages qui en sont la médiation instrumentale, les réalités «agglutinantes » qui prennent vie (Melé, 2008). Ils invitent encore à se déprendre de la mise en patrimoine des formes urbaines, sur un mode industriel et indifférencié, pour repenser notre compétence à édifier avec la ville passée (Choay, 2009). La pensée 
deleuzienne peut donc intéresser l'aménagement en ce qu'elle ne survalorise pas les formes géographiques existantes, ni ne prône la poursuite d'un futur qui les ignore. Elle nous situe dans un entre-deux, celui de l'action prudente à ce qui fait notre histoire et nous entoure, capable de s'ouvrir à la nouveauté par le moyen de l'expérimentation.

Cette réflexivité associée aux outils et méthodes de l'aménagement n’a d'autre but que de renouveler les façons d'interagir avec les milieux géographiques. Elle introduit donc à la question du sujet; non pas du sujet en général, pris comme une instance abstraite des réalités sociohistoriques, mais d'une écologie du sujet. Il s'agit, ensuite de préciser ce que le sujet doit au milieu et, rétablissant ce terme essentiel, mieux comprendre la place que l'aménagement peut accorder au sujet dès lors qu'il investit le milieu.

\section{Milieu et écologie du sujet}

Par l'usage non instrumental des normes qu'elle promeut, la pensée deleuzienne introduit, en aménagement, à une approche renouvelée des milieux géographiques. En particulier, en ouvrant la notion de milieu à une dimension de potentiel, elle découvre l'idée d'une écologie du sujet, selon laquelle le milieu géographique permet au sujet de se saisir comme tel et, partant, de raisonner sa façon d'habiter. Tels sont les deux points que nous développons à présent.

Si l'idée de potentiel est souvent associée à la notion de milieu, cette articulation reste souvent implicite et partiellement assumée. Dans les années 1970, la géographie humaniste a privilégié une lecture du milieu centrée sur l'intentionnalité humaine (Tuan, 2006) aux dépens d'un vécu dont la complexité réside aussi dans sa dimension matérielle (Entrikin, 1976). Plus récemment, Berque en a proposé une lecture plus globale: le milieu désigne autant les conditions de l'action que ses produits et la façon dont ceux-ci nous affectent en retour (Berque, 2000a). Il est à la fois empreinte et matrice. Cependant, sa critique de la civilisation technicienne, sous-tendue par l'idée d'un rapport originel au milieu dont il faudrait retrouver les codes (Berque, 2000 b), soulève certaines interrogations quant à la façon de penser la conduite de l'action. Comment approcher le milieu géographique selon des modes d'association plus ouverts?

Un moyen de progresser en ce sens consiste à asseoir la notion de milieu sur une pensée de l'émergence, telle que celle de Deleuze, parfois associé à Guattari (1980). Trois aspects retiennent notre attention:

- tout d'abord, la pensée de Deleuze nous permet d'ouvrir la notion de milieu à la question de la «multiplicité». Ceci consiste à approcher le milieu comme un réseau ouvert (c'est-à-dire le rhizome) dont les interactions donnent lieu à une grande diversité de combinaisons et d'agencements entre des entités hétérogènes, humaines et non humaines;

- ensuite, cette pensée invite à regarder positivement la «matérialité». Plutôt que d'être réduite au support passif des projets qui animent les hommes, la matérialité est la dimension qui fait du milieu un monde empirique et physique au sein duquel tous les corps s'affectent mutuellement. Il s'agit donc d'appréhender la compatibilité de nos modes d'existence en fonction de la qualité de leurs relations, de leurs attachements; 
- enfin, Deleuze nous permet d'augmenter la notion de milieu d'une réflexion sur le temps. Dans son œuvre, les milieux - au pluriel - sont codés, formés, signifiés. Ils renvoient, dans notre champ, aux milieux géographiques constitués dans le temps de l'histoire (Chronos), dont les formes évoluent selon des rapports de causalité. Le milieu - au singulier désigne un point de passage entre plusieurs milieux (Deleuze et Guattari, 1980; Deleuze, 1988). Il renvoie au sens commun du milieu comme entredeux. Alors que les milieux - au pluriel - sont les lieux de reproduction des formes et de leurs codages, le milieu - au singulier - nous situe à un niveau préformel, dans un temps plus exploratoire et novateur (Aiôn) à même de porter la question de l'émergence.

Le milieu géographique (multiplicité, matérialité, histoire/nouveauté) est donc pour notre usage une instance ductile ${ }^{5}$ où se reprécisent constamment les rapports entre les choses - ce qui lui confère une dimension de potentiel. Cette définition du milieu n'est pas centrée sur l'intentionnalité du sujet. Elle présente même des éléments de proximité avec les travaux de la sociologie des sciences et techniques (Latour, 1999) consistant à envisager une pensée du social élargie aux non-humains bien qu'elle ne s'y superpose pas complètement. Notre intérêt pour un cadre spatial nous conduit à donner un rôle non négligeable aux formes géographiques constituées dans le temps de l'histoire, sans souscrire, suivant en cela Descola (2005), à l'idée que le social se réduirait à une juxtaposition de collectifs se faisant et se défaisant en fonction des problématiques affrontées.

Adossée à une pensée de l'émergence, la notion de milieu découvre la figure du sujet dans sa plus fragile actualité. L'homme ne vit pas seulement dans un milieu, il vit avec ce milieu, et ne cesse de devenir à travers lui. Le milieu est ce qui permet au sujet de se saisir comme tel et de participer à l'instanciation d'un lieu à soi. Le sujet a lieu. Cet «avoir lieu» est une émergence. Il faut à l'homme beaucoup d'écoute et d'imagination pour donner à ses états relationnels et sensibles une qualification qui lui permettra de prendre part aux enjeux de son époque comme sujet.

Le sujet est souvent décrit comme une direction de conscience donnant forme et sens au monde. Notre orientation se distingue de cette logique du prédicat selon laquelle le sujet se donnerait le milieu «en tant que» situation de loisir, de travail, etc. Certes, le rôle du sujet est considérable dans l'instanciation d'un lieu à soi, mais nous ne pouvons prendre le risque de penser le lieu à l'image du sujet. C'est pourquoi, nous plaçons la question du devenir au fondement de celle de l'habiter: le sujet a lieu, il ne cesse de devenir avec et à travers son milieu.

Ainsi abordée, la notion de milieu donne accès à un «avoir lieu» du sujet plus vital qu'existentiel. Le sujet expérimente, par son corps et son expérience sensible, des possibilités de vie qui sont, pour lui, non déductibles et non intentionnelles. Prenant l'exemple des ambiances urbaines (Labussière, 2009b), nous avons suggéré qu'elles faisaient suite à des moments imprévus d'éclatement de la synthèse perceptive chez le sujet, initiant ce dernier à une découverte de l'urbain à un niveau préformel. La question de la prise en charge de ces processus non intentionnels est également forte

5 Ce caractère ductile, illustratif de la capacité du milieu à faire émerger du nouveau, place notre réflexion sur l'aménagement en amont du vocable plus courant d'aménagement du territoire, où l'action dialoguerait d'emblée avec les éléments stables de la nation. 
en aménagement (Soubeyran, 2007). Il ne s'agit pas de «faire monde» avec tout ou, à l'inverse, d'évacuer tout élément porteur d'incertitude. La question de la prudence est donc cruciale, pour l'aménagement comme pour le sujet, en ce qu'elle renvoie l'un comme l'autre à sa capacité de composer avec le milieu.

En ce sens, nous parlerions volontiers d'une écologie du sujet. Le sujet a lieu parce que son rapport au milieu lui permet de se saisir comme tel. Cette idée est présente chez Kant. Le sujet kantien est un "sujet sensible» (Benoist, 1996) défini par sa capacité d'être affecté. L'espace et le temps font de la sensibilité une façon d'être immédiatement au dehors qui ouvre l'espace d'un dedans : extériorité et intériorité se constituent par l'affection. C'est là un thème perceptible chez Kant, repris par Deleuze: le sujet comme «plissement du dehors» (2004: 115). Le rapport au monde est constitutif d'un rapport à soi générateur d'une subjectivité.

Ce legs kantien entre en résonance chez Deleuze avec la question du milieu, saisie comme un potentiel. Dans le temps de l'histoire, le sujet ne cesse de se donner une nouvelle actualité en puisant dans la virtualité d'un Moi perpétuellement affecté de quoi inventer les formes de son existence. Cette aptitude à poser la question de sa propre actualité, à en constituer le sens pour soi, est une entreprise significative de la modernité (Foucault, 1994), qui n'est pas contradictoire avec une pensée du devenir (Deleuze, 2004). Le sujet trouve dans la relation au milieu un soubassement primordial pour se saisir comme sujet et s'établir. Cela suggère que l'habiter n'est pas le seul fait d'une condition terrestre, un séjour parmi les choses, mais la forme du devenir: à la fois la position du sujet (le sujet a lieu) et les formes que celui-ci invente pour occuper cette position (le lieu du sujet) ${ }^{6}$.

Dans sa dimension de potentiel, la notion de milieu tisse un lien essentiel entre l'aménagement et la logique du sujet. Il y a en effet, du point de vue symptomatologique, une identité de structure entre l'exploration du milieu par l'aménagement et l'effort du sujet pour constituer un rapport au monde comme rapport à soi. Dans l'un et l'autre cas, le rapport au sensible est décisif pour explorer le milieu, donner une place aux processus non intentionnels, expérimenter en-deçà des normes des modes d'existence partagés. La transition énergétique contemporaine, abordée par l'entremise du cas de l'énergie éolienne, est un contexte intéressant pour analyser ces interactions entre l'aménagement, le milieu et la logique du sujet.

\section{Transition énergétique et prospective du milieu: l'exemple des planifications éoliennes}

En rapprochant l'aménagement d'une activité clinique, nous disposons d'un cadre heuristique pour analyser des situations sous contrainte, par exemple dans le cas de recompositions territoriales et paysagères en contexte de transition énergétique et de lutte contre le réchauffement climatique. Par leur importance, ces recompositions appellent à revisiter le diagnostic en aménagement et invitent à étudier les modalités d'un pilotage selon des valeurs émergentes.

6 Nous invitons le lecteur à se reporter aux travaux de Berdoulay et Entrikin (1998) pour un approfondissement des thèmes du lieu et du sujet. 
La politique éolienne française est un cas exemplaire d'une politique environnementale issue de réflexions à l'échelle globale et suscitant des recompositions profondes de nos territoires et de nos paysages. Si la politique énergétique française est traditionnellement centralisée, le cas éolien préfigure des enjeux qui relèvent davantage d'une décentralisation énergétique (Nadaï, 2007).

Par sa forte présence visuelle et le caractère modulable de ses implantations, l'énergie éolienne introduit dans des espaces souvent reculés, encore peu affectés par de grandes infrastructures, des enjeux d'aménagement et de paysage tout à fait nouveaux. Plus encore, son gigantisme ne permet plus de raisonner en termes de limitation des impacts. L'éolien met donc fortement en cause les pratiques et les méthodes de l'aménagement et les défie d'accompagner des processus politiques et sociaux complexes dont l'enjeu est d'aboutir à des recréations territoriales et paysagères partagées.

Ces mutations changent le rapport de l'aménagement aux milieux géographiques en ce qu'elles incitent à passer d'une logique de gestion des impacts à une visée de projet. Mais elles posent aussi la question du sujet et de sa place dans ces processus. L'éolien nous rappelle que notre électricité vient de quelque part (Pasqualetti, 2000). Il remet au premier plan notre responsabilité quant aux conséquences de nos modes de production et de consommation énergétiques. Il s'agit de rendre nos choix en matière de politique énergétique compatibles avec nos politiques de gestion des paysages, de préservation de la nature, de conservation du patrimoine, etc. L'émergence de nouveaux paysages de l'énergie bouscule nos modes d'habiter et implique de nouvelles façons de nous tenir comme sujets vis-à-vis d'un sensible qui nous lie plus que jamais aux enjeux du local et du global.

L’aménagement peut jouer un rôle crucial en ce sens et participer à la gouvernance de la transition énergétique à condition qu'il renouvelle ses méthodes et les normes qui les sous-tendent. En France, la politique de gestion du paysage s'est historiquement structurée autour de valeurs scéniques et visuelles lui conférant le statut d'un bien commun national. La bonne administration de ces valeurs repose sur leur traduction dans des figures géométriques valant un zonage de protection. Par sa présence dans le paysage, l'éolien bouscule cette politique paysagère centralisée, fortement normée et adossée à une logique territoriale (dedans/hors du zonage).

Inaudibles du point de vue des zonages et des règlements qu'elles bousculent, ces mutations paysagères appellent en quelque sorte une autre "écoute», une pensée de l'aménagement à même de saisir ce brouhaha du sensible, de lui donner des formes qui le rendent collectivement négociable sans pour autant le figer dans des modalités trop instrumentales.

La pertinence du modèle clinique est qu'il démultiplie la question du sensible et offre une grande amplitude de moyens pour se pencher sur les processus informels qui travaillent l'aménagement. Grâce à lui, nous pouvons effectuer un déplacement de la question du sensible des termes de la relation (l'aménagement/le milieu) à ce qui les relie pour en faire l'objet même de l'analyse. Cela redispose la façon d'étudier la prise en compte du milieu par l'aménagement : le sensible ne renvoie plus au registre de l'expérience, mais à une catégorie englobante qui porte l'analyse au devant de différents jeux relationnels qui codent et donnent forme aux rapports entre l'aménagement et le milieu. Ce faisant, nous parvenons à articuler trois niveaux d'analyse: 
- le niveau cognitif, où il s’agit de comprendre la façon dont l'aménagement, par ses connaissances, ses modes de représentation et leur mise en œuvre, configure la question du sensible (comme le paysage), c'est-à-dire définit le domaine de ce qui est à connaître et distribue, entre les acteurs, la capacité d'y prendre part ou non;

- le niveau politique, où il s'agit de comprendre la façon dont la planification partage le sensible ${ }^{7}$; en effet, la façon dont l'espace est mis en représentation, puis ces représentations en circulation entre les différents acteurs, produit des occasions de mise en partage qui tendent à définir par défaut le public auquel elles s'adressent et, donc, à exclure des formes de sensibilité et d'expérience (Labussière, 2009a);

- le niveau de l'expérience, où il s'agit d'appréhender le sensible comme une instance profondément ductile où l'émergence de nouveaux jeux relationnels entre les choses (l'échelle éolienne, par exemple) met à l'épreuve les réseaux d'humains et de non-humains, ainsi que les catégories de pensée et d'action de l'aménagement.

Nous avons là trois jeux relationnels qui permettent de caractériser la question du sensible en aménagement et, partant, d'analyser les modalités de renouvellement de ses modèles d'action et de ses normes. Quelques expériences de planifications éoliennes dans le cas français peuvent illustrer notre propos.

En Aveyron, les services de l'État ont élaboré dans l'urgence, faute de doctrine au niveau national, une planification éolienne par accumulation de contraintes réglementaires (Nadaï et Labussière, 2009). Cette planification oriente par défaut l'implantation des projets éoliens dans les délaissés patrimoniaux et produit, ce faisant, des contre-performances paysagères, des covisibilités inattendues avec des monuments protégés ou encore des effets de surdensité. En réduisant la logique paysagère à une logique zonale, cette planification reste corsetée par des représentations issues d'une tradition administrative de gestion centralisée du paysage. La valeur normative de ces représentations les rend difficilement négociables et limite l'exploration du potentiel éolien à une logique territoriale (dedans/hors du zonage) qui occulte l'exploration du potentiel de chaque situation.

Dans l'Aude, la Ligue de protection des oiseaux (LPO) a renouvelé ses méthodes d'observation des oiseaux afin de négocier la mise en site des projets avec les développeurs éoliens (Nadaï et Labussière, 2010). Au lieu des traditionnels comptages de migrateurs, la méthode du micro-siting s'intéresse aux comportements des oiseaux et à leurs stratégies de franchissement des éoliennes (bifurcation, traversée, survol, plongeon). La LPO rassemble des comportements analogues entre espèces, crée des familles de vol, différencie l'espace selon des microvoies de passage et donne une mesure du degré de risque pour les oiseaux qui les empruntent. Les documents d'aménagement s'en trouvent renouvelés grâce à un vocabulaire cartographique qui valorise les capacités d'adaptation des oiseaux à l'éolien.

7 Nous reprenons la notion de «partage du sensible» à Rancière (2000) - pour son emploi en aménagement (Labussière, 2009a). 
Les élus du Parc naturel régional de la Narbonnaise ont pour leur part entrepris un processus de planification innovante confié à un cabinet de paysage (Labussière et Nadaï, 2011). L'originalité de cette planification éolienne tient à un dispositif iconographique judicieux qui lui a permis de mobiliser la diversité des échelles convoquées par l'éolien (le site, le grand paysage) sans jamais céder à la facilité de représentations cartographiques qui réduiraient les négociations à une logique territoriale (dedans/hors du zonage). Ceci a permis aux élus du parc d'élaborer une planification concertée (associations, développeurs, services de l'État) visant à situer des potentiels éoliens futurs sans les indexer de façon définitive au territoire. La prise en compte des logiques de situation dès la phase amont (dialogue entre les échelles) permet à cette planification de porter des préconisations paysagères qui seront par la suite reprises et développées par les promoteurs éoliens en phase de projet.

Ces différents cas d'étude mettent l'accent sur la capacité de la planification à se «ramifier », c'est-à-dire à rouvrir les systèmes normatifs qui la sous-tendent (droit des sols, classifications paysagères, avifaunes...) pour les indexer à des logiques de situation, de sorte que l'application de la norme se mue en une intelligence négociée de son usage donnant droit de cité à un plus grand nombre d'humains et de non-humains. $\mathrm{Si}$, dans le premier cas, cette mise en réseau échoue, en revanche les deux suivants montrent qu'elle est permise grâce à un travail important sur le potentiel relationnel des formes cartographiques.

L'importance de cette mise en réseau est qu'elle peut être porteuse de réciprocité entre des réalités sensibles non commensurables. Le renouvellement de la représentation en aménagement est crucial pour explorer une pluralité d'espace-temps en donnant au représenté (le paysage, l'oiseau, l'habitant...) la capacité de circuler entre la logique de l'action, avec ses exigences normatives, et la logique de la situation qui le dote de compétences et d'un potentiel d'adaptation à la nouveauté. Le sensible est donc au cœur des méthodes et des pratiques de l'aménagement puisque sa capacité à expérimenter des représentations ramifiées lui permet d'accéder à une position stratégique, d'écoute et d'exploration d'une pluralité de jeux relationnels situés qui conditionnent l'efficacité de ses arbitrages.

Un autre atout de cette approche par le sensible est qu'elle nous permet d'identifier des formes d'apprentissage non «pures» en aménagement, c'est-à-dire sans raisonner les cas d'étude en termes d'échec ou d'innovation. Par exemple, la planification éolienne d'Eure-et-Loir (Nadaï et Labussière, 2011) est innovante par la façon dont elle renouvelle ses représentations du paysage pour rendre discutable avec les développeurs des sensations visuelles problématiques sur le terrain (densité, respiration, contraste, intimité...) ; mais elle est plutôt conservatrice en ce que l'administration cartographie ces sensations paysagères à petite échelle, avec peu de références au territoire, pour se prémunir contre d'éventuelles revendications habitantes quant aux orientations adoptées. La question du sensible permet donc d'analyser l'émergence de nouvelles pratiques planificatrices et de cerner leur degré de normativité d'après la façon dont elles agencent les dimensions cognitive, politique et expérientielle. 


\section{Conclusion}

Cet article pose la question du sensible en aménagement en tentant de voir en quoi, au-delà du registre de l'expérience, elle pourrait contribuer à réfléchir sur les normes de ses modèles d'action, leurs points de blocage et d'innovation.

Notre analyse suggère que la façon dont le sensible (cognitif, politique, expérientiel) est configuré et distribué décide en partie de la normativité de la démarche aménagiste et de son ouverture à la logique du sujet. L'expérimentation de planifications «ramifiées», dans le domaine de l'énergie éolienne, est un cas exemplaire de renégociation de notre rapport au sensible à la fois tel que nous le vivons, tel que nous le constituons en objet à connaître et à protéger, et tel que nous le mettons en politique.

Ce pilotage selon des valeurs émergentes en aménagement peut utilement être rapproché d'une pensée clinique où les manifestations sensibles, non encore constituées, nous renvoient à nos ignorances et nous forcent à expérimenter des instrumentations et des codes pour nous mettre en position d'écoute et d'exploration de la multiplicité. Ceci renvoie somme toute à une intelligence prospective qui n'est plus celle d'un exercice sur le futur, au sens classique, mais celle d'un exercice sur le devenir, en ce qu'il engage une attention directe pour les milieux géographiques comme potentiel - ce qui situe l’aménagement du côté d’une prospective du milieu.

Ce positionnement théorique, permis par une lecture nuancée de la pensée de Deleuze, offre enfin une position intéressante pour discuter la façon dont fonctionne la critique de la modernité en aménagement. Cette dernière, portée notamment par les approches collaboratives, consiste en grande partie à définir les "bons » mécanismes intégrateurs (du social, de l'environnement...), ce qui tend à les instituer et les pérenniser comme des "extériorités » à l'aménagement. La critique est donc toute relative et mériterait d'être davantage ouverte à l'étude des processus porteurs de réciprocités entre l'aménagement, les milieux géographiques et le sujet. Notre approche ouvre des pistes dans ce sens en ce qu'elle suggère que toute démarche d'aménagement, même parmi les plus hiérarchiques, engage un rapport au sensible (cognitif, politique, expérientiel), mais que peu de ces démarches savent organiser ces réciprocités et évaluer le parti qu'elles pourraient en tirer. 


\section{Bibliographie}

AMPHOUX, Pascal, THIBAUD Jean-Paul et CHELKOFF Grégoire (dir.) (2004) Ambiances en débat. Bernin, À la croisée.

ANTONIOLI, Manola (2003) Géophilosophie de Deleuze et Guattari. Paris, L'Harmattan.

AUGOYARD, Jean-François (1995) L'environnement sensible et les ambiances architecturales. L'Espace géographique, $\mathrm{n}^{\circ} 4$, p. 302-319.

BENOIST, Jocelyn (1996) Kant et les limites de la synthèse. Le sujet sensible. Paris, Presses universitaires de France.

BENOIST, Jocelyn et MERLINI Fabio (2001) Historicité et spatialité. Le problème de l'espace dans la pensée contemporaine. Paris, Vrin.

BERDOULAY, Vincent (2000) Le milieu, entre description et récit. De quelques difficultés d'une approche de la complexité. Dans Vincent Berdoulay et Olivier Soubeyran (dir.) Milieu, colonisation et développement durables: perspectives géographiques sur l'aménagement. Paris, L'Harmattan, p. 25-37.

BERDOULAY, Vincent et ENTRIKIN, J. Nicholas (1994) Singularité des lieux et prospective. Espaces et sociétés, n ${ }^{\text {os }} 74-75$, p. 189-201.

BERDOULAY, Vincent et ENTRIKIN, J. Nicholas (1998) Lieu et sujet: perspectives théoriques. L'Espace géographique, no2, p. 111-121.

BERLEANT, Arnold (1992) The aesthetics of the environment. Philadelphia, Temple University Press.

BERQUE, Augustin (2000a) Ecoumène. Introduction à l'étude des milieux humains. Paris, Belin.

BERQUE Augustin (dir.) (2000b) Logique du lieu et dépassement de la modernité. Tomes 1 et 2. Ousia, Librairie philosophique Vrin.

BLANC, Nathalie (2008) Vers une esthétique environnementale. Paris, Quæ.
BLONDEL, Éric (1986) Nietzsche, le corps et la culture. La philosophie comme généalogie philologique. Paris, Presses universitaires de France.

BUCHANAN, Ian et LAMBERT, Gregg (dir.) (2006) Deleuze and Space. Édimbourg, Edinburgh University Press.

BUYDENS, Mireille (2005) [1990] Sahara. L'esthétique de Gilles Deleuze. Paris, Vrin.

CHOAY, Françoise (1980) La règle et le modèle. Sur la théorie de l'architecture et de l'urbanisme. Paris, Seuil.

CHOAY, Françoise (2009) Le patrimoine en questions. Anthologie pour un combat. Paris, Seuil.

COSGROVE, Denis (1984) Social formation and symbolic landscape. London, Croom Helm.

DE GRAMONT, Jérôme (1996) Kant et la question de l'affectivité. Lecture de la troisième critique. Paris, Vrin.

DELEUZE, Gilles (1962) Nietzsche et la philosophie. Paris, Presses universitaires de France.

DELEUZE, Gilles (1973) Cours de Vincennes du 14 mai 1973 sur le Corps sans organes. Webdeleuze. [En ligne.] http://www. webdeleuze.com.

DELEUZE, Gilles (1988) Le Pli. Paris, Les Éditions de Minuit.

DELEUZE, Gilles (1997) [1963] La philosophie critique de Kant. Paris, Presses universitaires de France.

DELEUZE, Gilles (2003) [1981] Spinoza. Philosophie pratique. Paris, Les Éditions de Minuit.

DELEUZE, Gilles (2004) [1986] Foucault. Paris, Les Éditions de Minuit.

DELEUZE, Gilles (2006) [1964] Proust et les signes. Paris, Presses universitaires de France.

DELEUZE, Gilles et GUATTARI Félix (1980) Capitalisme et schizophrénie 2. Mille plateaux. Paris, Les Éditions de Minuit. 
DESCOLA, Philippe (2005) Par-delà nature et culture. Paris, Gallimard.

DEWITTE, Jacques (2001) Pays paysage: à propos d'une difficulté théorique de l'artialisation. Dans Françoise Chenet, Michel Collot et Baldine Saint-Girons (dir.) Le paysage, état des lieux. Bruxelles, Éditions Ousia, p. 419-440.

DEWSBURY, John-David (2000) Performativity and the event: enacting a philosophy of difference. Environment and Planning D: Society and Space, no ${ }^{\circ}$, p.473-496.

DOEL, Marcus (1993) Proverbs for paranoids: writing geography on hollowed ground. Transactions of the Institute of British Geographers, no 18 , p. 377-394

DOEL, Marcus (1996) A hundred thousand lines of flight: a machinic introduction to the nomad thought and scrumpled geography of Gilles Deleuze and Félix Guattari. Environment and Planning D: Society and Space, no 14, p. 421-439.

DOEL, Marcus (1999) Poststructuralist geographies. The diabolical art of spatial science. Édimbourg, Edinburgh University Press.

ENTRIKIN, J. Nicholas (1976) Contemporary humanism in geography. Annals of the Association of American Geographers, vol. 66, no 4 , p. 615-632.

EPD (1992) Geography and postmodernism, Environment and Planning D: Society and Space, vol. 10, no 2 .

EPD (1996) Environment and Planning D: Society and Space, vol. 14, n० 4 .

FABUREL, Guillaume (2003) Le bruit des avions. Facteur de révélation et de construction de territoires. L'Espace géographique, no3, p. 205-223.

FOUCAULT, Michel (1994) Qu'est-ce que les Lumières? Dans Dits et écrits, 1954-1988. Paris, Gallimard, p. 679-688.

FOUCAULT, Michel (2007) [1963] Naissance de la clinique. Paris, Presses universitaires de France.

GALE, Stephen et OLSSON, Gunnar (1979) Philosophy and geography. London, Reidel Publishing Company.
HUTIN, Jean-François (2006) L'examen clinique à travers l'histoire. Paris, Éditions Glyphe.

KUPFER, Joseph (2003) Engaging nature aesthetically. Journal of aesthetic education, vol. 37, no 1 , p. 77-89.

LABUSSIÈRE, Olivier (2009a) Les stratégies esthétiques dans la contestation des projets d'aménagement: le milieu géographique entre singularité et exception. L'information géographique, vol.73, nº 2 , p. 68-88.

LABUSSIÈRE, Olivier (2009b) Éléments pour une symptomatologie des ambiances urbaines. Articulo - Journal of Urban Research [En ligne], Hors-série 2, «Esthétiques et pratiques des paysages urbains».

LABUSSIÈRE, Olivier (à paraître) Pensée aménagiste, pensée clinique. La figure du médecin-musicien. Actes du Colloque Musique, territoire et développement local.

LABUSSIÈRE, Olivier et NADAÏ, Alain (2011) Expérimentations cartographiques et devenirs paysagers. La planification éolienne de la Narbonnaise. Espaces et Sociétés, no 146.

LAENNEC, René Théophile Hyacinte (1819) De l'auscultation médiate ou traité du diagnostic des maladies des poumons et du cœur, fondé principalement sur ce nouveau moyen d'exploration. [En ligne.] http://www.archive.org/details/delauscultationm01aen

LATOUR, Bruno (1999) Politiques de la nature. Comment faire entrer les sciences en démocratie? Paris, La Découverte.

LE CORBUSIER (1994) [1925] Urbanisme. Paris, Champs Flammarion.

LOLIVE, Jacques et SOUBEYRAN, Olivier (2007) L'émergence des cosmopolitiques. Paris, La Découverte.

MELE, Patrice (2008) Territoires d'action et qualifications de l'espace. Dans Patrice Melé et Corrine Larrue (dir.) Territoires d'action. Paris, L'Harmattan, p. 15-44. 
NADAÏ, Alain (2007) Planning, Siting and the local Acceptance of Wind Power: Some Lessons from the French Case. Energy Policy, n³5, p. 2715-2726.

NADAÏ, Alain et LABUSSIÈRE, Olivier (2009) Windpower planning in France (Aveyron); from state regulation to local planning. Land Use Policy, vol.26, no3, p. 744-754.

NADAÏ, Alain et LABUSSIÈRE, Olivier (2010) Birds, wind and the making of windpower landscapes in Aude, Southern France. Landscape Research, vol. 35, n² p. 209-233.

NADAÏ, Alain et LABUSSIÈRE, Olivier (à paraître) Walking the field, recomposing a visual landscape: planning wind power in the Eure-et-Loir.

PASQUALETTI, Martin (2000) Morality, space and the power of wind-energy landscapes. The Geographical Review, vol.90, n³, p. 381-394.

PEET, Richard (2006) Modern geographical thought. London, Wiley, Blackwell.

RANCIÈRE, Jacques (2000) Le partage du sensible. Esthétique et politique. Paris, La Fabrique Éditions.

ROGER, Alain (1997) Court traité du paysage. Paris, Gallimard.

SAUVAGNARGUES, Anne (2005) Deleuze et l'art. Paris, Presses universitaires de France.

SCHLANGER, Judith (1971) Les métaphores de l'organisme. Paris, Vrin.

SOUBEYRAN, Olivier (2000) De la prise en compte du milieu à son évacuation dans la géographie médicale du début du XX siècle. Dans Vincent Berdoulay et Olivier Soubeyran (dir.) Milieu, colonisation et développement durable. Paris, L'Harmattan, p. 101-116.

SOUBEYRAN, Olivier (2007) Pensée aménagiste et tautologies. Dans Jacques Lolive et OIivier Soubeyran (dir.) L'émergence des cosmopolitiques. Paris, La Découverte, p. 125-153.

THRIFT, Nigel (1996) Spatial formations. Londres, Sage Publications.
THRIFT, Nigel (2000) Afterwords. Environment and planning D: Society and Space, vol. 18, n², p. 213-255.

THRIFT, Nigel et DEWSBURY, John-David (2000) Dead geographies - and how to make them live. Environment and Planning D: Society and Space, vol. 18, no 4 , p. 411- 432 .

TUAN, Yi-Fu (2006) [1976] Espace et lieu: la perspective de l'expérience. Gollion, Infolio. 